\title{
Gemcitabine and Carboplatin Combination Chemotherapy for Elderly Patients with Advanced Non-small Cell Lung Cancer: A Feasibility Study
}

\author{
Young Jin Yuh, M.D., Hyo Rak Lee, M.D. and Sung Rok Kim, M.D. \\ Department of Internal Medicine, College of Medicine, Inje University, Sanggye Paik Hospital, Seoul, Korea
}

Purpose: Although platinum based chemotherapy is known to improve the survival duration for the patients with non-small cell lung cancer, the role of platinum for elderly patient is not yet clear. We administered gemcitabine and carboplatin combination therapy to elderly patients with NSCLC. The aim of this study was to evaluate the efficacy and toxicities of this regimen for elderly patients.

Materials and Methods: The eligibility criteria were as follows: pathologically confirmed NSCLC, an age $\geq 65$ years, advanced disease with stage IIIB or IV and the patients were chemotherapy-naive. The treatment regimen was as follows; gemcitabine $1,000 \mathrm{mg} / \mathrm{m}^{2}$ was administered on days 1 and 8 and carboplatin $A U C=5$ was administered on day 1 . This regimen was repeated every 3 weeks. The efficacy was evaluated in terms of the response rate, the time to progression and the overall survival duration.

Results: From Dec 2001 to Feb 2005, a total of 20 pa-

\section{INTRODUCTION}

Lung cancer is the leading cause of cancer death in most countries (1), including South Korea (2). Lung cancer is literally a kind of geriatric disease and more than two-thirds of lung cancer deaths occur in persons who are over 65 years of age (3).

A meta-analysis revealed that patients with advanced non-small cell lung cancer (NSCLC) and who were treated with platinum-containing chemotherapy regimens had a $10 \%$ improvement of their 1 year survival compared with those who were managed with supportive care only (4). However, most randomized trials are usually carried out on highly selected patients such as young patients. Elderly patients have been

Correspondence: Young Jin Yuh, Department of Internal Medicine, College of Medicine, Inje University, Sanggye Paik Hospital, 761-1, Sanggye 7-dong, Nowon-gu, Seoul 139-707, Korea. (Tel) 82-2-950-1460, (Fax) 82-2-950-1955, (E-mail) yjyuh@ paik.ac.kr Received May 23, 2008, Accepted July 23, 2008

This work was supported by a 2003 Inje University research grant. tients were entered into this study. The median patient age was 68 years (range: $65 \sim 75$ ). 19 patients were evaluable for their treatment response. A partial response was obtained in 8 patients (response rate: $42.1 \%, 95 \%$ Cl: $19.4 \sim 64.8 \%$ ). The median time to progression and the survival duration were 136 days and 453 days, respectively. Among a total of 65 cycles of treatment, grade 3 or 4 leukopenia and thrombocytopenia were observed in $7.7 \%$ and $13.9 \%$ of the cycles, respectively. Grade 3 or 4 vomiting was observed in $7.7 \%$ of the cycles. Grade 3 skin rash developed in $1.5 \%$ of the cycles. 1 patient died of septic shock after chemotherapy.

Conclusions: Gemcitabine and carboplatin combination chemotherapy was relatively safe and effective for treating elderly patients with NSCLC. (Cancer Res Treat. 2008;40:116-120)

Key Words: Gemcitabine, Carboplatin, Non-small cell lung cancer, Aged

excluded from most clinical trials because of the concern over excess toxicity (5).

Some reports have recently clarified the benefit of chemotherapy for elderly patients. A randomized trial of vinorelbine versus the best supportive care for patients 70 years of age or older demonstrated a definite improvement in the overall survival rate and quality of life with administering chemotherapy (6). Using instrumental variable propensity analysis, chemotherapy for NSCLC seems to be effective for elderly patients with an efficacy that is similar to that of the randomized trials on mostly younger, highly selected patients (7). The above data suggest that chemotherapy must be considered for treating elderly patients with advanced NSCLC.

Nevertheless, the most appropriate regimen for elderly patients with NSCLC has not yet been established. The exact role of platinum compounds and combination chemotherapy for elderly patient is still being debated.

Gemcitabine is active against NSCLC and it is well tolerated too. Gemcitabine monotherapy in elderly patients with NSCLC produced an overall response rate of $22.2 \sim 38.5 \%$ with minimal toxicities $(8,9)$. The response rate of $22.2 \%$ is comparable to that of $20.0 \%$ of the Elderly Lung Cancer Vinorelbine Italian Study (ELVIS) trial (6).

Platinum combination chemotherapy is considered as a standard 
therapy for patients with NSCLC. However, many medical oncologists often hesitate to add cisplatin to treat elderly patients because of the concern about excessive toxicities.

Carboplatin is less toxic than cisplatin and it has been demonstrated to be just as efficacious for the treatment of NSCLC (10). A previous report suggests that the gemcitabine and carboplatin combination chemotherapy may be superior to gemcitabine single therapy in terms of the response rate and overall survival with comparable non-hematologic toxicities (11).

There are various doses and schedules for gemcitabine and carboplatin combination therapy. However, the efficacy and toxicity seem to be similar among the various methods (12).

On the basis of these considerations, we administered gemcitabine and carboplatin combination therapy to elderly Korean patients with NSCLC, and we report here on the results.

\section{MATERIALS AND METHODS}

\section{1) Eligibility}

The patients with an age $\geq 65$ had to fulfill all the following criteria to be eligible for this study: have a histologically or cytologically proven NSCLC, have stage IIIB or IV disease that wasn't suitable for curative resection or recurred disease after resection, have measurable lesions and a good performance status (ECOG $0 \sim 2$ ), have no experience of previous chemotherapy with or without a previous history of radiotherapy history, have adequate bone marrow (granulocyte $\geq 1,800$ / $\mathrm{mm}^{3}$, platelet $\geq 100,000 / \mathrm{mm}^{3}$ ), hepatic (total bilirubin $\leq 2.0$ $\mathrm{mg} / \mathrm{dL}$ ) and, renal (creatinine clearance $\geq 50 \mathrm{~mL} / \mathrm{min}$ ) functions. An informed written consent was obtained from all the patients who were entered into this study.

\section{2) Treatment schedule}

Gemcitabine was administrated at a dose of $1,000 \mathrm{mg} / \mathrm{m}^{2}$ on days 1 and 8. Carboplatin was administered at a dose of AUC $=5$ on day 1 . The dose of carboplatin was calculated according to the Calvert formula. This combined regimen was repeated every 3 weeks.

The dose of gemcitabine on day 8 was modified as follows according to the CBC. It was reduced to $50 \%$ of the planned dose if the granulocyte count was 500 to $999 / \mathrm{mm}^{3}$ and/or the platelet count was 50,000 to $74,900 / \mathrm{mm}^{3}$. The administration of gemcitabine was omitted if the granulocyte count was below $500 / \mathrm{mm}^{3}$ or the platelet count was below $50,000 / \mathrm{mm}^{3}$.

This treatment was repeated until disease progression or the appearance of unacceptable toxicities or until a maximum 4 cycles. After the planned treatment, further treatment with this protocol or second line treatment were performed based on the physician's choice.

\section{3) Treatment evaluation}

A CBC, blood chemistry tests, chest X-rays and physical exam were repeated before every chemotherapy cycle. The follow-up studies with computerized tomography and bone scanning were performed every 2 cycles of chemotherapy. The responses were classified according to the World Health Organization criteria. Treatment toxicities were evaluated according to the National Cancer Institute Common Toxicity Criteria version 3.0.

\section{4) Statistical considerations}

The primary object of this study was response rate. The secondary endpoints were the overall survival duration, the time to progression and the toxicities of this regimen. The overall survival duration is defined as the time between the date of starting treatment and the last date that the patient was known to be alive. Progression free survival is defined as the time from the date of starting treatment to disease progression or to death from disease progression or unknown causes. Both overall survival and the time to progression were calculated using the Kaplan-Meier method. The Medcalc program version 8.0 was used for statistical analysis.

\section{RESULTS}

\section{1) Patient's characteristics}

From Dec 2001 to Feb 2005, a total of 20 patients were entered into this study. Thirteen $(65 \%)$ patients were male and seven $(35 \%)$ were female. Three patients (15\%) had stage IIIB disease and $17(85 \%)$ had stage IV disease. The performance status was $6(30 \%), 12(60 \%)$ and $2(10 \%)$ patients in ECOG 0,1 and 2, respectively. Eight patients $(40 \%)$ were (70 years of age and the median patient age was 68 years (range: 65 75). The patients' characteristics are summarized on Table 1.

\section{2) Treatment received}

Twenty patients received a total of 65 cycles of chemotherapy. The patients received $1 \sim 6$ cycles of treatment (median: 4 cycles). Twelve patients $(60 \%$ of the total patients) fulfilled the planned therapy of 4 or more cycles ( 6 patients were aged less than 70 and 6 patients were aged 70 or more). The number of cycles of chemotherapy received was not significantly different between these two age groups $(\mathrm{p}=0.225)$. Among the total 65 cycles of therapy, the dose at day 8 was reduced in 10 cycles $(15.4 \%)$.

Table 1. Patient's characteristics

\begin{tabular}{lcc}
\hline & No & $\%$ \\
\hline No. patients entered & 20 & \\
Sex. male/female & $13 / 7$ & $65 / 35$ \\
Age & 12 & 60 \\
$\quad 65 \sim 69$ & 8 & 40 \\
$\quad \geq 70$ & & \\
ECOG performance status & 6 & 30 \\
0 & 12 & 60 \\
1 & 2 & 10 \\
2 & & \\
Histology & 11 & 55 \\
$\quad$ Squamous cell carcinoma & 8 & 40 \\
Adenocarcinoma & 1 & 5 \\
Undifferentiated carcinoma & & \\
Stage & 2 & 10 \\
IIIB & 18 & 90 \\
IV & & \\
\hline
\end{tabular}


118 Cancer Res Treat. 2008;40(3)

Table 2. Responses after treatment

\begin{tabular}{lrr}
\hline & No & $\%$ \\
\hline Complete remission & 0 & 0.0 \\
Partial remission & 8 & 42.1 \\
Stable disease & 9 & 47.4 \\
Progressive disease & 2 & 10.5 \\
\hline Total & 19 & 100.0 \\
\hline
\end{tabular}

\section{3) Response}

Among the total 20 patients, 19 patients were evaluable for their treatment response, except the 1 case of early death. There was no complete remission. Partial remissions were observed in 8 patients among the total 19 patients $(42.1 \%)$. So, the overall response rate was $42.1 \%$ (95\% C.I.: $19.4 \sim 64.8 \%$ ). The responses after treatment are summarized in Table 2 . The response rates for the patients with an age $<70$ year and an age $\geq 70$ were $50 \%$ and $25 \%$, respectively. The difference of the response rate was not significant between the younger and older groups $(\mathrm{p}=0.51)$.

\section{4) The time to progression and the duration of overall survival}

The median time to progression was 136 days (Fig. 1) and the median duration of overall survival was 453 days (Fig. 2). The median duration of overall survival for the patients with an age $<70$ and that of the patients with an age $\geq 70$ were 337 days and 453 days, respectively. The duration of overall survival was not different between the two age groups $(p=0.89)$. The median duration of overall survival for the responders to chemotherapy and the non-responders were 542 days and 327 days, respectively. The difference between the responders and non-responders was not significantly different, either $(p=0.163)$.

\section{5) Toxicities}

Toxicities were evaluated for a total 65 cycles of treatment. One patient aged 67 years died of pneumonia and the consequent septic shock without neutropenia after 1 cycle of chemotherapy. This patient experienced severe nausea and vomiting after chemotherapy, and the suspected cause of the pneumonia was aspiration during vomiting.

The grade 3 or 4 hematologic and non-hematologic toxicities are presented in Table 3. Two patients refused further treatment after 2 cycles of treatment. These 2 patients experienced grade 3 vomiting and grade 3 skin rash, respectively. Hematologic toxicities of grade 4 were rare (the incidence of neutropenia and thrombocytopenia were $6.2 \%$ and $6.2 \%$, respectively).

\section{6) Second line treatment}

12 patients among the total 20 patients received second line treatment after failure with gemcitabine and carboplatin combination chemotherapy. Most of them (11 of 12) were treated with a docetaxel-based regimen. 1 patient was treated with gefitinib.

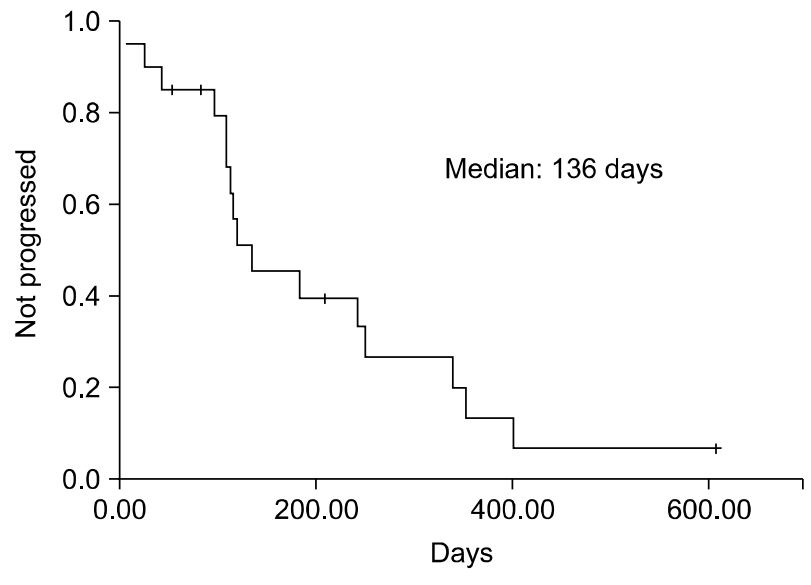

Fig. 1. Time to progression.

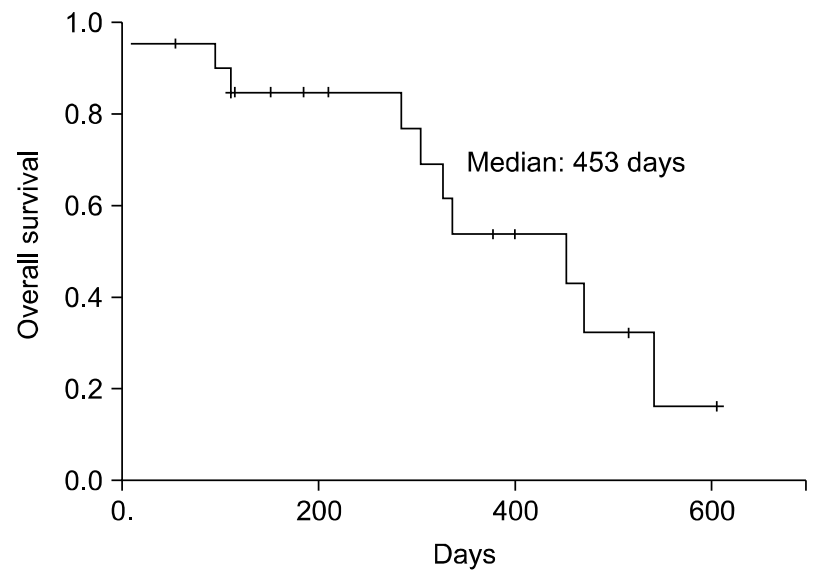

Fig. 2. Overall survival.

Table 3. Toxicities ( $\%$ of cycles) in total 65 cycles of treatment

\begin{tabular}{lll}
\hline & Grade 3 & Grade 4 \\
\hline Hematologic & & \\
Leukopenia & $5(7.7)$ & $0(0.0)$ \\
Neutropenia & $3(4.6)$ & $4(6.2)$ \\
Thrombocytopenia & $5(7.7)$ & $4(6.2)$ \\
Non-hematologic & & \\
Nausea & $2(3.1)$ & $1(1.5)$ \\
Vomiting & $4(6.2)$ & $1(1.5)$ \\
Diarrhea & $1(1.5)$ & $0(0.0)$ \\
Mucositis & $1(1.5)$ & $0(0.0)$ \\
Skin rash & $1(1.5)$ & $0(0.0)$ \\
\hline
\end{tabular}

\section{DISCUSSION}

For the patients with NSCLC, the 3rd generation platinumbased regimens yield similar results in terms of the response rate and the overall survival duration. All of the 3rd generation 
platinum-based regimens are now considered as standard regimens for NSCLC (13). Among them, gemcitabine and platinum combination chemotherapy has been one of the most popular regimens. The reported response rates of gemcitabine plus cisplatin for treating NSCLC are about $40 \%$ (14 16). The regimen with gemcitabine plus carboplatin (substituted for cisplatin) may result in a slightly inferior response rate, but the overall survival duration is very similar to that for the gemcitabine plus cisplatin regimen (17). In fact, many believe that carboplatin and cisplatin have equivalent activities for NSCLC, while carboplatin has a more favorable non-hematologic toxicity profile (18).

In this study, the response rate of $42.1 \%$ is comparable to the reports that included younger age patients $(11,13)$. This response rate is superior to that of vinorelbine single therapy in the ELVIS (Elderly Lung Cancer Vinorelbine Italian Study Group) trial (6). The median duration of overall survival for our study was 453 days. It also seems encouraging when compared with the median duration of overall survival (196 days) for vinorelbine single therapy (6), even if the duration of overall survival is also dependent upon the second line therapy.

There were some differences in study design between this study and the ELVIS trial. Most importantly, we defined the elderly group as patients aged 65 or more, while the ELIVS trial included only patients aged 70 or more. There is currently no consensus upon the definition of an elderly patient Some authors have enrolled patients aged 65 or more in their study for elderly patients (19), while others have enrolled only patients aged 70 or more (20). In this report, the response rate of the patients aged less than 70 was higher than that of the patients aged 70 or more, even though the difference was not statistically significant. The relatively small number of patients in each age group could have caused in this statistically insignificant difference in the 2 groups' response rate. Therefore, we could not exclude the possibility that this small study population resulted in more favorable outcomes. Moreover, most of the patients in this study had a good performance status. A good performance status is generally related with a good response rate, as well as a longer overall duration of survival.

For the aspect of the toxicity profile, this regimen was well-tolerated with only rare grade 3 or 4 hematologic and non-hematologic toxicities. However, two patients refused further treatment after 2 cycles of treatment. This seems to be caused by the attitude of the elderly patients and their family members toward the medical treatment. They were not willing to endure the toxicities and they too easily gave up on further treatment. The clinical benefit of chemotherapy for elderly patients has been displayed by many reports (20), and we should more seriously recommend chemotherapy for this group of patients. These efforts could possibly change the attitude of elderly patients and their family members.

The toxicities of chemotherapy may depend on the race of the patients. Traditionally, the Japanese treat their patients with less intensive doses of chemotherapy in the belief that an Asian cannot tolerate the dose that's defined for the Caucasian (21). However, in this study, even elderly Asian people could tolerate the platinum containing chemotherapy.

\section{CONCLUSION}

Gemciatine and carboplatin combination chemotherapy was tolerated and effective for elderly patients with NSCLC.

\section{REFERENCES}

1. Parkin DM. Global cancer statistics in the year 2000. Lancet Oncol. 2001;2:533-43.

2. Kim SY, Ryoo HM, Bae SH, Kim KC, Hyun DS, Lee SC, et al. Efficacy of administration of weekly docetaxel combined with platinum as a first-line treatment for patients with advanced non-small cell lung cancer. Korea J Med. 2007;72:625-31.

3. Gridelli C, Perrone F, Monfardini S. Lung cancer in the elderly. Eur J Cancer. 1997;33:2313-4.

4. Non-small Cell Lung Cancer Collaborative Group. Chemotherapy in non-small cell lung cancer: a meta-analysis using updated data on individual patients from 52 randomised clinical trials. BMJ. 1995;311:899-909.

5. Hutchins LF, Unger JM, Crowley JJ, Coltman CA, Albain KS. Underrepresentation of patients 65 years of age or older in cancer-treatment trials. N Engl J Med. 1999;341:2061-7.

6. The Elderly Lung Cancer Vinorelbine Italian Study Group. Effects of vinorelbine on quality of life and survival of elderly patients with advanced non-small-cell lung cancer. J Natl Cancer Inst. 1999;91:66-72.

7. Earle CC, Tsai JS, Gelber RD, Weinstein MC, Neumann PJ, Weeks JC. Effectiveness of chemotherapy for advanced lung cancer in the elderly: instrumental variable and propensity analysis. J Clin Oncol. 2001;19:1064-70.

8. Ricci S, Antonuzzo A, Galli L, Tibaldi C, Bertuccelli M, Lopes Pegna A, et al. Gemcitabine monotherapy in elderly patients with advanced non-small cell lung cancer: a multicenter phase II study. Lung Cancer. 2000;27:75-80.

9. Bianco V, Rozzi A, Tonini G, Santini D, Magnolfi E, Vincenzi $\mathrm{B}$, et al. Gemcitabine as single agent chemotherapy in elderly patients with stages III-IV non-small cell lung cancer (NSCLC): a phase II study. Anticancer Res. 2002;22:3053-6.

10. Klastersky J, Sculier JP, Lacroix H, Dabouis G, Bureau G, Libert $\mathrm{P}$, et al. A randomized study comparing cisplatin or carboplatin with etoposide in patients with advanced nonsmall-cell lung cancer: European Organization for Research and Treatment of Cancer Protocol 07861. J Clin Oncol. 1990; 8:1556-62.

11. Sederholm C, Hillerdal G, Lamberg K, Kolbeck K, Dufmats $\mathrm{M}$, Westberg R, et al. Phase III trial of gemcitabine plus carboplatin versus single-agent gemcitabine in the treatment of locally advanced or metastatic non-small-cell lung cancer: the Swedish Lung Cancer Study Group. J Clin Oncol. 2005;23: 8380-8.

12. Masters GA, Argiris AE, Hahn EA, Beck JT, Rausch PG, Ye $\mathrm{Z}$, et al. A randomized phase II trial using two different treatment schedules of gemcitabine and carboplatin in patients with advanced non-small-cell lung cancer. J Thorac Oncol. 2006;1:19-24.

13. Schiller JH, Harrington D, Belani CP, Langer C, Sandler A, Krook J, et al. Eastern Cooperative Oncology Group. Comparison of four chemotherapy regimens for advanced non-small-cell lung cancer. N Engl J Med. 2002;346:92-8.

14. Iaffaioli RV, Tortoriello A, Facchini G. Phase I-II study of gemcitabine and carboplatin in Stage IIIB-IV non-small cell 
lung cancer. J Clin Oncol. 1999;17:921-6.

15. Rinaldi M, Crino L, Scagliotti GV, Mosconi AM, De Marinis F, Gridelli C, et al. A three-week schedule of gemcitabinecisplatin in advanced non-small-cell lung cancer with two different cisplatin dose levels: a phase II randomized trial. Ann Oncol. 2000;11:1295-300.

16. Cardenal F, Lopez-Cabrerizo MP, Anton A, Alberola V, Massuti B, Carrato A, et al. Randomized phase III study of gemcitabine-cisplatin versus etoposide-cisplatin in the treatment of locally advanced or metastatic non-small-cell lung cancer. J Clin Oncol. 1999;17:12-8.

17. Mazzanti P, Massacesi C, Rocchi MB, Mattioli R, Lippe P, Trivisonne $\mathrm{R}$, et al. Randomized, multicenter, phase II study of gemcitabine plus cisplatin versus gemcitabine plus carboplatin in patients with advanced non-small cell lung cancer. Lung Cancer. 2003;41:81-9.
18. Go RS, Adjei AA. Review of the comparative pharmacology and clinical activity of cisplatin and carboplatin. J Clin Oncol. 1999; 17:409-22.

19. Hainsworth JD, Burris HA 3rd, Litchy S, Morrissey LH, Barton $\mathrm{JH}$, Bradof JE, et al. Weekly docetaxel in the treatment of elderly patients with advanced nonsmall cell lung carcinoma. Cancer. 2000;89:328-33.

20. Gridelli C, Aapro M, Ardizzoni A, Balducci L, De Marinis F, Kelly K, et al. Treatment of advanced non-small-cell lung cancer in the elderly: results of an international expert panel. J Clin Oncol. 2005;23:3125-37.

21. Gridelli C, Aapro M, Ardizzoni A, Balducci L, De Marinis F, Kelly K, et al. Treatment of advanced non-small-cell lung cancer in the elderly: results of an international expert panel. J Clin Oncol. 2005;23:3125-37. 